\title{
On the tubular surfaces in $E^{3}$
}

\author{
Ali Cakmak ${ }^{1}$ and Omer Tarakci ${ }^{2}$ \\ ${ }^{1}$ Department of Mathematics, Faculty of Arts and Sciences, Bitlis Eren University, Bitlis, Turkey \\ ${ }^{2}$ Department of Mathematics, Faculty of Science, Ataturk University, Erzurum, Turkey
}

\begin{abstract}
In this study, we obtain surfaces at a constant distance from the edge of regression on a tubular surface indicated by $M^{f}$, condition that $M$ is denoted by a tubular surface in $E^{3}$. Firstly, we show that $M^{f}$ is a tubular surface, for $\lambda_{1}=0$. Then, we calculate curvatures of $M^{f}$ and find some relationships between curvatures of surfaces $M$ and $M^{f}$. Finally, we research curvatures of center curve of $M^{f}$, for some special cases.
\end{abstract}

Keywords: Surfaces at a constant distance from the edge of regression on a surface, Tubular surfaces, Geodesic curve, Asymptotic curve, Line of curvature.

\section{Introduction}

First of all, Tarakcı and Hacısalihoğlu are the first ones to introduce surfaces at a constant distance from the edge of regression on a surface in $E^{3}$ in 2002[1]. Actually, these surfaces were based on the study which is curve at a constant distance from the edge of regression on a curve proposed by Hans Vogler in 1963. They obtained surfaces at a constant distance from the edge of regression on a surface in $E^{3}$ taking a surface instead of a curve and calculated for these surfaces some properties and theorems known for parallel surfaces [2]. Later, conjugate tangent vectors and asymptotic directions for these surfaces are given in[3]. Euler theorem and Dupin indicatrix for these surfaces are given in[4]. In 2010, Sağlam and Kalkan obtained the some theorems and properties for surfaces at a constant distance from edge of regression on a surface in $E_{1}^{3}$ Minkowski 3-space[5]. The same authors examined Euler theorem and Dupin indicatrix for these surfaces in $E_{1}^{3}[6]$. Also the same authors studied conjugate tangent vectors and asymptotic directions for these surfaces in $E_{1}^{3}$ [7]. In 2014, Yurttançikmaz and Tarakc1 investigated the relationship between focal surfaces and surfaces at a constant distance from the edge of regression on a surface [8]. Surface at a constant distance from the edge of regression on a surface of revolution in $E^{3}[9]$ and the image curves on surfaces at a constant distance from the edge of regression on a surface of revolution are given in[10] In relation to the concept of curvature, in recent times some authors studied Riemannian metric $g$ and curvature tensor field $R$ of Riemannian manfolds on tangent bundle[11, 12,13].

In differential geometry, tubular surfaces are one of the subjects that are studied extensively since tubular surfaces are among the surfaces which are easier to describe both analytically and kinematically. Recently, the studies on the tubular surfaces are given in $[14,15,16,17]$. Generally, a tubular surface generated by constructing a tube around a circle is known as a torus. The purpose of this paper is to introduce, analyze and compare tubular surfaces and surfaces at a constant distance from the edge of regression on a tubular surface in $E^{3}$. Let $M$ be a tubular surface and $M^{f}$ be surface at a constant distance from the edge of regression on tubular surface $M$. We show that if $\lambda_{1}=0, M^{f}$ is a tubular surface. Furthermore, we calculate Gauss and mean curvatures, first and second fundamental forms for $M^{f}$. Then, we obtain 
these curvatures for some special cases and we show relationships between curvatures of the surfaces $M$ and $M^{f}$. Finally, we give the conditions of being geodesic curve, asymptotic curve and line of curvature of center curve of tubular surface.

\section{Preliminaries}

Definition 1. Let $M$ and $M^{f}$ be two surfaces in $E^{3}$ and $N_{P}$ be a unit normal vector at a point $P$ of surface $M$. Let $T_{P} M$ be tangent space at $P \in M$ and $\left\{X_{P}, Y_{P}\right\}$ be an orthonormal bases of $T_{P} M$. Take a unit vector $Z_{P}=d_{1} X_{P}+d_{2} Y_{P}+d_{3} N_{P}$, where $d_{1}, d_{2}, d_{3} \in R$ are constant numbers and $d_{1}^{2}+d_{2}^{2}+d_{3}^{2}=1$. If there is a fonction $f$ defined by, $f: M \rightarrow M^{f}, f(P)=P+r Z_{P}$, $r$ constant, then the surface $M^{f}$ is called the surface at a constant distance from the edge of regression on $M . M$ and $M^{f}$ are shown by the pair $\left(M, M^{f}\right)$.If $d_{1}=d_{2}=0$, then we have $Z_{P}=N_{P}$ and so $M$ and $M^{f}$ are parallel surfaces.

Now, we represent parameterization of surfaces at a constant distance from the edge of regression on $M$. Let $(\phi, U)$ be a parameterization of $M$

$$
\begin{gathered}
\phi: U \subset E^{2} \rightarrow M \\
(u, v) \quad \phi(u, v)
\end{gathered}
$$

In this case, $\left\{\phi_{u}, \phi_{v}\right\}$ is bases vector of $T_{P} M$. Let $N_{P}$ be a unit normal vector at a point $P$ and $d_{1}, d_{2}, d_{3} \in R$ be constant numbers then we can write that $Z_{P}=d_{1} \phi_{u}+d_{2} \phi_{v}+d_{3} N_{P}$, where $\phi_{u}$ and $\phi_{v}$ are, respectively, partial derivatives of $\phi$ with respect to $u$ and $v$. Since $M^{f}=\left\{f(P): f(P)=P+r Z_{P}\right\}$, a parametric representation of $M^{f}$ is

$$
\psi(u, v)=\phi(u, v)+r Z(u, v) .
$$

Thus, we obtain

$$
M^{f}=\left\{\psi(u, v): \psi(u, v)=\phi(u, v)+r\left(d_{1} \phi_{u}(u, v)+d_{2} \phi_{v}(u, v)+d_{3} N(u, v)\right\} .\right.
$$

If we take $r d_{1}=\lambda_{1}, r d_{2}=\lambda_{2}, r d_{3}=\lambda_{3}$, we get

$$
M^{f}=\left\{\psi(u, v): \psi(u, v)=\phi(u, v)+\lambda_{1} \phi_{u}(u, v)+\lambda_{2} \phi_{v}(u, v)+\lambda_{3} N(u, v), \lambda_{1}^{2}+\lambda_{2}^{2}+\lambda_{3}^{2}=r^{2}\right\}
$$

Calculation of $\psi_{u}$ and $\psi_{v}$ gives us that

$$
\begin{gathered}
\psi_{u}=\phi_{u}+\lambda_{1} \phi_{u u}+\lambda_{2} \phi_{v u}+\lambda_{3} N_{u}, \\
\psi_{v}=\phi_{v}+\lambda_{1} \phi_{u v}+\lambda_{2} \phi_{v v}+\lambda_{3} N_{v} .
\end{gathered}
$$

Here, $\phi_{u u}, \phi_{u v}, \phi_{v u}, N_{u}, N_{v}$ are calculated as like as[1]. If parameter curves are line of curvatures of $M$ and let $u$ and $v$ be arc lenght of these line of curvatures, we have following equations.

$$
\begin{gathered}
\phi_{u u}=-\kappa_{1} N, \\
\phi_{v v}=-\kappa_{2} N, \\
\phi_{u v}=\phi_{v u}=0, \\
N_{u}=\kappa_{1} \phi_{u}, \\
N_{v}=\kappa_{2} \phi_{u} .
\end{gathered}
$$

From the eq. (1) and (2), we get

$$
\begin{gathered}
\psi_{u}=\left(1+\lambda_{3} \kappa_{1}\right) \phi_{u}-\lambda_{1} \kappa_{1} N, \\
\psi_{v}=\left(1+\lambda_{3} \kappa_{2}\right) \phi_{v}-\lambda_{2} \kappa_{2} N
\end{gathered}
$$


and $\left\{\psi_{u}, \psi_{v}\right\}$ be a bases of $\chi\left(M^{f}\right)$. The unit vector field $N^{f}$ of $M^{f}$ is

$$
N^{f}=\frac{\psi_{u} \times \psi_{v}}{\left\|\psi_{u} \times \psi_{v}\right\|}=\frac{\lambda_{1} \kappa_{1}\left(1+\lambda_{3} \kappa_{2}\right) \phi_{u}+\lambda_{2} \kappa_{2}\left(1+\lambda_{3} \kappa_{1}\right) \phi_{v}+\left(1+\lambda_{3} \kappa_{1}\right)\left(1+\lambda_{3} \kappa_{2}\right) N}{\sqrt{\lambda_{1}^{2} \kappa_{1}^{2}\left(1+\lambda_{3} \kappa_{2}\right)^{2}+\lambda_{2}^{2} \kappa_{2}^{2}\left(1+\lambda_{3} \kappa_{1}\right)^{2}+\left(1+\lambda_{3} \kappa_{1}\right)^{2}\left(1+\lambda_{3} \kappa_{2}\right)^{2}}}
$$

where, $\kappa_{1}, \kappa_{2}$ are principal curvatures of the surface $M$. If we take

$$
A=\sqrt{\lambda_{1}^{2} \kappa_{1}^{2}\left(1+\lambda_{3} \kappa_{2}\right)^{2}+\lambda_{2}^{2} \kappa_{2}^{2}\left(1+\lambda_{3} \kappa_{1}\right)^{2}+\left(1+\lambda_{3} \kappa_{1}\right)^{2}\left(1+\lambda_{3} \kappa_{2}\right)^{2}}
$$

we can write

$$
N^{f}=\frac{\lambda_{1} \kappa_{1}\left(1+\lambda_{3} \kappa_{2}\right)}{A} \phi_{u}+\frac{\lambda_{2} \kappa_{2}\left(1+\lambda_{3} \kappa_{1}\right)}{A} \phi_{v}+\frac{\left(1+\lambda_{3} \kappa_{1}\right)\left(1+\lambda_{3} \kappa_{2}\right)}{A} N
$$

If $\kappa_{1}=\kappa_{2}$ and $\lambda_{3}=-\frac{1}{\kappa_{1}}=-\frac{1}{\kappa_{2}}$, since $\psi_{u}$ and $\psi_{v}$ are not linear independent, so $M^{f}$ is not regular surface. We will not consider this case [1].

Definition 2. A tube of radius $r$ of a set $c$ is the set of points at a distance $r$ from $c$. In particular, if $c(s)$ is a regular space curve parameterized by arc length whose curvature does not vanish, then the normal vector $N$ and binormal vector $B$ are always perpendicular to $c$, and the circle $\cos \theta N(s)+\sin \theta B(s)$ is perpendicular to $c$ at $c(s)$. So as the circle moves around $c$, it traces out a tube, provided the tube radius $r$ is small enough so that the tube is not self-intersecting. We can parameterize this surface by

$$
\phi(s, \theta)=c(s)+r(\cos \theta N(s)+\sin \theta B(s))
$$

where $\theta \in[0,2 \pi)$. Here, $c(s)$ is called the center curve and $r$ is radius of tubular surface. [18].

Thus, by using the equation (3), we can easily calculate very important geometrical quantities. Then, from the expressions

$$
\begin{aligned}
& \phi_{\theta}=r(-\sin \theta N+\cos \theta B), \\
& \phi_{s}=(1-r \kappa \cos \theta) T+\tau \phi_{\theta}, \\
& \phi_{\theta \theta}=-r(\cos \theta N+\sin \theta B), \\
& \phi_{s \theta}=r \kappa \sin \theta T+\tau \phi_{\theta \theta}, \\
& \phi_{s s}=\left(-r \kappa^{\prime} \cos \theta+r \kappa \tau \sin \theta\right) T+\left(\kappa-r\left(\kappa^{2}+\tau^{2}\right) \cos \theta-r \tau^{\prime} \sin \theta\right) N+\left(-r \tau^{2} \sin \theta+r \tau^{\prime} \cos \theta\right) B,
\end{aligned}
$$

we obtain,

$$
\begin{gathered}
K=\frac{e g-f^{2}}{E G-F^{2}}=\frac{-\kappa \cos \theta}{r(1-r \kappa \cos \theta)}, \\
H=\frac{e G-2 f F+g E}{2\left(E G-F^{2}\right)}=\frac{1}{2}\left(\frac{1}{r}+K r\right),
\end{gathered}
$$

and principal curvatures,

$$
\begin{gathered}
\kappa_{1}=\frac{-\kappa \cos \theta}{1-r \kappa \cos \theta}, \\
\kappa_{2}=\frac{1}{r} .
\end{gathered}
$$




\section{Main results}

We know that tubular surface $M$ of radius $r$ around a set $c$ is the set of points at a distance $r$ from $c$, such that $c: I \rightarrow E^{3}$, a regular unit speed space curve. Let $P$ be a point on $M$ and $\{T, N, B\}$ be Frenet frame at $P \in M$. We have,

$$
P=c(s)+r(\cos \theta N(s)+\sin \theta B(s))
$$

for $P \in M$. Thus, we can write

$$
\phi: I \times[0,2 \pi) \rightarrow E^{3}, \quad \phi(s, \theta)=c(s)+r(\cos \theta N(s)+\sin \theta B(s))
$$

Hence, we have $\phi(I \times[0,2 \pi))=M$.

Now, we represent parameterization of surface $M^{f}$ at a constant distance from the edge of regression on tubular surface. Let $M$ be a tubular surface in $E^{3}$ and $n_{P}$ be a unit normal vector and $T_{P} M$ be tangent space at point $P$ of surface $M$ and $\left\{\left.\phi_{S}\right|_{P},\left.\phi_{\theta}\right|_{P}\right\}$ be an orthogonal bases of $T_{P} M$. Therefore, from the eq. (3) if we take partial derivative with respect to $s$ and $\theta$, we have

$$
\begin{gathered}
\phi_{s}=c^{\prime}(s)+r\left(\cos \theta N^{\prime}(s)+\sin \theta B^{\prime}(s)\right), \\
\phi_{s}(s, \theta)=(1-r \kappa \cos \theta) T-r \tau \sin \theta N+r \tau \cos \theta B \\
\left\|\phi_{s}\right\|=\sqrt{1-2 r \kappa \cos \theta+r^{2} \kappa^{2} \cos ^{2} \theta+r^{2} \tau^{2}}=A, \\
\phi_{\theta}(s, \theta)=-r \sin \theta N+r \cos \theta B .
\end{gathered}
$$

Furthermore, we define unit vectors $X$ and $Y$ as

$$
\begin{gathered}
X=\frac{\phi_{s}}{\left\|\phi_{s}\right\|}=\frac{1-r \kappa \cos \theta}{A} T-\frac{r \tau \sin \theta}{A} N+\frac{r \tau \cos \theta}{A} B \\
Y=\frac{\phi_{\theta}}{\left\|\phi_{\theta}\right\|}=-\sin \theta N+\cos \theta B
\end{gathered}
$$

and the unit normal vector $n$ as

$$
n=\frac{\phi_{s} \times \phi_{\theta}}{\left\|\phi_{s} \times \phi_{\theta}\right\|}=-\cos \theta N-\sin \theta B .
$$

Thus, we get parameterization of surface $M^{f}$ at a constant distance from the edge of regression on tubular surface as follows:

$$
\begin{gathered}
\psi(s, \theta)=\phi(s, \theta)+\lambda_{1} X+\lambda_{2} Y+\lambda_{3} n \\
\psi(s, \theta)=c(s)+\frac{\lambda_{1}}{A}(1-r \kappa \cos \theta) T+\left(r \cos \theta-\frac{r \tau \lambda_{1}}{A} \sin \theta-\lambda_{2} \sin \theta-\lambda_{3} \cos \theta\right) N \\
+\left(r \sin \theta+\frac{r \tau \lambda_{1}}{A} \cos \theta+\lambda_{2} \cos \theta-\lambda_{3} \sin \theta\right) B
\end{gathered}
$$

Also, we write surface $M^{f}$ at a constant distance from the edge of regression on tubular surface $M$

$$
\begin{gathered}
f: M \rightarrow M^{f}, \\
M^{f}=\left\{\psi(s, \theta)=\phi(s, \theta)+\lambda_{1} X+\lambda_{2} Y+\lambda_{3} n, \lambda_{1}=\text { const. }, \lambda_{2}=\text { const } ., \lambda_{3}=\text { const } .\right\} .
\end{gathered}
$$


Theorem 1. Let $\phi(s, \theta)$ be a parameterization of tubular surface $M$ in $E^{3}$, such that $\phi(s, \theta)=c(s)+r(\cos \theta N(s)+\sin \theta B(s))$. Then, $M^{f}$ is a tubular surface if and only if $\lambda_{1}=0$.

Proof. If the equation (9) is a tubular surface $\frac{\lambda_{1}}{A}(1-r \kappa \cos \theta)=0$, where $1-r \kappa \cos \theta \neq 0$ since $\phi(s, \theta)$ is a regular tubular surface. For this reason $\lambda_{1}=0$. Then, if the value $\lambda_{1}=0$ is written instead of $\lambda_{1}$ in the equation (9), we have

$$
\psi(s, \theta)=c(s)+\left(\left(r-\lambda_{3}\right) \cos \theta-\lambda_{2} \sin \theta\right) N+\left(\left(r-\lambda_{3}\right) \sin \theta+\lambda_{2} \cos \theta\right) B .
$$

By multiplying both sides of the eq. (10) with $\frac{\sqrt{\left(r-\lambda_{3}\right)^{2}+\lambda_{2}^{2}}}{\sqrt{\left(r-\lambda_{3}\right)^{2}+\lambda_{2}^{2}}}$, we have

$$
\begin{aligned}
\psi(s, \theta)=c(s) & +\left(\frac{r-\lambda_{3}}{\sqrt{\left(r-\lambda_{3}\right)^{2}+\lambda_{2}^{2}}} \cos \theta-\frac{\lambda_{2}}{\sqrt{\left(r-\lambda_{3}\right)^{2}+\lambda_{2}^{2}}} \sin \theta\right) N \sqrt{\left(r-\lambda_{3}\right)^{2}+\lambda_{2}^{2}} \\
& +\left(\frac{r-\lambda_{3}}{\sqrt{\left(r-\lambda_{3}\right)^{2}+\lambda_{2}^{2}}} \sin \theta+\frac{\lambda_{2}}{\sqrt{\left(r-\lambda_{3}\right)^{2}+\lambda_{2}^{2}}} \cos \theta\right) B \sqrt{\left(r-\lambda_{3}\right)^{2}+\lambda_{2}^{2}} .
\end{aligned}
$$

By taking,

$$
\frac{r-\lambda_{3}}{\sqrt{\left(r-\lambda_{3}\right)^{2}+\lambda_{2}^{2}}}=\cos \beta
$$

and

$$
\frac{\lambda_{2}}{\sqrt{\left(r-\lambda_{3}\right)^{2}+\lambda_{2}^{2}}}=\sin \beta
$$

we get,

$$
\psi(s, \theta)=c(s)+((\cos \theta \cos \beta-\sin \theta \sin \beta) N+(\sin \theta \cos \beta+\cos \theta \sin \beta) B) \sqrt{\left(r-\lambda_{3}\right)^{2}+\lambda_{2}^{2}}
$$

and if we admit as

$$
\begin{aligned}
& \cos \theta \cos \beta-\sin \theta \sin \beta=\cos (\theta+\beta)=\cos \gamma \\
& \sin \theta \cos \beta+\cos \theta \sin \beta=\sin (\theta+\beta)=\sin \gamma
\end{aligned}
$$

and

$$
\sqrt{\left(r-\lambda_{3}\right)^{2}+\lambda_{2}^{2}}=R=\text { const }
$$

we have

$$
\psi(s, \gamma)=c(s)+R(\cos \gamma N(s)+\sin \gamma B(s))
$$

Hence, we obtain that $M^{f}$ is a new tubular surface. Conversely, if $\lambda_{1}=0$, we get easily the equation (18) again. Thus, the proof is completed.

Example 1. We consider that

$$
\phi(s, \theta)=\left((1-\cos \theta) \cos \frac{s}{5 \sqrt{2}}+\frac{7}{5 \sqrt{2}} \sin \frac{s}{5 \sqrt{2}} \sin \theta,(1-\cos \theta) \sin \frac{s}{5 \sqrt{2}}-\frac{7}{5 \sqrt{2}} \sin \theta \cos \frac{s}{5 \sqrt{2}}, \frac{7}{5 \sqrt{2}} s+\frac{\sin \theta}{5 \sqrt{2}}\right)
$$



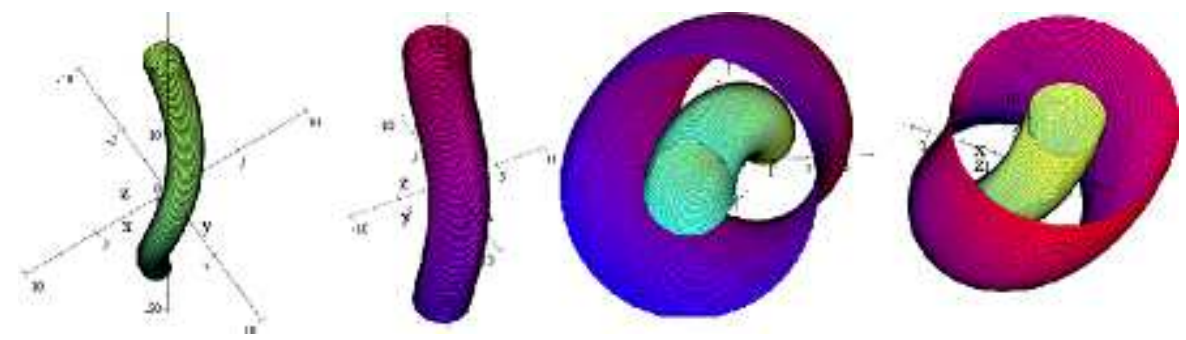

Fig. 1: Tubular surface and surfaces at a constant distance from the edge of regression on tubular surface.

where unit speed curve $c(s)=\left(\cos \frac{s}{5 \sqrt{2}}, \sin \frac{s}{5 \sqrt{2}}, \frac{7}{5 \sqrt{2}} s\right)$ and $r=1$. According to this, surface at a constant distance from the edge of regression on $\phi(s, \theta)$ under the condition that $\lambda_{1}=0$ as follows:

$$
\psi(s, \theta)=\left(\begin{array}{c}
\cos \frac{s}{5 \sqrt{2}}\left(1-\cos \theta+\lambda_{2} \sin \theta+\lambda_{3} \cos \theta\right)+\frac{7}{5 \sqrt{2}} \sin \frac{s}{5 \sqrt{2}}\left(\sin \theta+\lambda_{2} \cos \theta-\lambda_{3} \sin \theta\right), \\
\sin \frac{s}{5 \sqrt{2}}\left(1-\cos \theta+\lambda_{2} \sin \theta+\lambda_{3} \cos \theta\right)-\frac{7}{5 \sqrt{2}} \cos \frac{s}{5 \sqrt{2}}\left(\sin \theta+\lambda_{2} \cos \theta-\lambda_{3} \sin \theta\right), \\
\frac{7}{5 \sqrt{2}} s+\frac{1}{5 \sqrt{2}}\left(\sin \theta+\lambda_{2} \cos \theta-\lambda_{3} \sin \theta\right)
\end{array}\right)
$$

Now, considering that under the condition that $\lambda_{1}=0$, we calculate I, II. fundamental forms and curvatures of surface at a constant distance from the edge of regression on a tubular surface in $E^{3}$. From the equation (18), we know that $\psi(s, \gamma)=c(s)+R(\cos \gamma N(s)+\sin \gamma B(s))$ is parameterization of surface at a constant distance from the edge of regression on a tubular surface in $E^{3}$. Here, by taking

$$
\begin{gathered}
\psi_{\gamma}(s, \gamma)=-R \sin \gamma N+R \cos \gamma B \\
\psi_{s}(s, \gamma)=(1-R \kappa \cos \gamma) T-R \tau \sin \gamma N+R \tau \cos \gamma B \\
\psi_{s}(s, \gamma)=(1-R \kappa \cos \gamma) T+\tau \psi_{\gamma}(s, \gamma),
\end{gathered}
$$

where $\psi_{s}, \psi_{\gamma}$ are basis vectors at a point $f(P)$ of $M^{f}$, we have

$$
n^{f}=\frac{\psi_{s} \times \psi_{\gamma}}{\left\|\psi_{s} \times \psi_{\gamma}\right\|}=-\cos \gamma N-\sin \gamma B
$$

where $n^{f}$ is unit normal vector of surface at a constant distance from the edge of regression on tubular surface. Then, first fundamental form coefficients are obtained as follows:

$$
\begin{gathered}
E=\left\langle\psi_{s}, \psi_{s}\right\rangle=(1-R \kappa \cos \gamma)^{2}+R^{2} \tau^{2}, \\
F=\left\langle\psi_{s}, \psi_{\gamma}\right\rangle=R^{2} \tau, \\
G=\left\langle\psi_{\gamma}, \psi_{\gamma}\right\rangle=R^{2}
\end{gathered}
$$

In addition, second fundamental form coefficients are calculated as follows:

$$
\begin{gathered}
\psi_{s s}=\left(-R \kappa^{\prime} \cos \gamma+R \kappa \tau \sin \gamma\right) T+\left(\kappa-R\left(\kappa^{2}+\tau^{2}\right) \cos \gamma-R \tau^{\prime} \sin \gamma\right) N+\left(-R \tau^{2} \sin \gamma+R \tau^{\prime} \cos \gamma\right) B, \\
\psi_{s \gamma}=R \kappa \sin \gamma T+\tau \psi_{\gamma \gamma}=\psi_{\gamma s},
\end{gathered}
$$




$$
\begin{gathered}
\psi_{\gamma \gamma}=-R(\cos \gamma N+\sin \gamma B), \\
e=\left\langle\psi_{s s}, n^{f}\right\rangle=-\kappa \cos \gamma(1-R \kappa \cos \gamma)+R \tau^{2}, \\
f=\left\langle\psi_{s \gamma}, n^{f}\right\rangle=R \tau, \\
g=\left\langle\psi_{\gamma \gamma}, n^{f}\right\rangle=R .
\end{gathered}
$$

Hence, Gauss curvature $K^{f}$ and mean curvature $H^{f}$ at an arbitrary point of the surface $M^{f}$ are found as

$$
\begin{gathered}
K^{f}=\frac{e g-f^{2}}{E G-F^{2}}=-\frac{\kappa \cos \gamma}{R(1-R \kappa \cos \gamma)}, \\
H^{f}=\frac{e G-2 f F+g E}{2\left(E G-F^{2}\right)}=\frac{1}{2}\left(\frac{1}{R}+R K^{f}\right) .
\end{gathered}
$$

In this case, principal curvatures of $M^{f}$ are

$$
\begin{gathered}
\kappa_{1}^{f}=-\frac{\kappa \cos \gamma}{(1-R \kappa \cos \gamma)}, \\
\kappa_{2}^{f}=\frac{1}{R} .
\end{gathered}
$$

Now, we investigate $M^{f}$ for $\lambda_{1}=0$. From the equation (3), we have

$$
\phi(s, \theta)=c(s)+r(\cos \theta N(s)+\sin \theta B(s)) .
$$

Curvatures of tubular surface are given by the eq. (4), (5), (6) and (7). From these equations, we get,

$$
\begin{gathered}
\cos \theta=\frac{K r}{\kappa\left(K r^{2}-1\right)}, \\
\sin \theta=\frac{\sqrt{K^{2} r^{2}\left(\kappa^{2} r^{2}-1\right)-\kappa^{2}\left(2 \kappa r^{2}-1\right)}}{\kappa\left(K r^{2}-1\right)} .
\end{gathered}
$$

From the equations (12), (13), (15), (17), (26), (30) and (31), Gauss curvature $K^{f}$ of $M^{f}$ is

$$
K^{f}=\frac{-\kappa\left(\frac{r-\lambda_{3}}{R} \frac{K r}{\kappa\left(K r^{2}-1\right)}-\frac{\lambda_{2}}{R} \frac{\sqrt{K^{2} r^{2}\left(\kappa^{2} r^{2}-1\right)-\kappa^{2}\left(2 \kappa r^{2}-1\right)}}{\kappa\left(K r^{2}-1\right)}\right)}{R\left(1-R \kappa\left(\frac{r-\lambda_{3}}{R} \frac{K r}{\kappa\left(K r^{2}-1\right)}-\frac{\lambda_{2}}{R} \frac{\sqrt{K^{2} r^{2}\left(\kappa^{2} r^{2}-1\right)-\kappa^{2}\left(2 \kappa r^{2}-1\right)}}{\kappa\left(K r^{2}-1\right)}\right)\right)} .
$$

If $r=\frac{1}{\kappa_{2}}$ is used, we obtain

$$
K^{f}=\frac{-K \kappa_{2}\left(1-\lambda_{3} \kappa_{2}\right)+\lambda_{2} \kappa_{2}^{2} \sqrt{\kappa^{2}\left(\kappa_{1}-\kappa_{2}\right)^{2}-K^{2}}}{\left(\left(1-\lambda_{3} \kappa_{2}\right)^{2}+\lambda_{2}^{2} \kappa_{2}^{2}\right)\left(\lambda_{3} K-\kappa_{2}+\lambda_{2} \sqrt{\kappa^{2}\left(\kappa_{1}-\kappa_{2}\right)^{2}-K^{2}}\right)} .
$$


Similarly, if $R=\sqrt{\left(r-\lambda_{3}\right)^{2}+\lambda_{2}^{2}}, r=\frac{1}{\kappa_{2}}$ and $K^{f}$ are written at the equation (27), we find

$$
H^{f}=\frac{1}{2}\left(\frac{-K-\kappa_{2}^{2}+2 \kappa_{2}\left(\lambda_{3} K+\lambda_{2} \sqrt{\kappa^{2}\left(\kappa_{1}-\kappa_{2}\right)^{2}-K^{2}}\right)}{\sqrt{\left(1-\lambda_{3} \kappa_{2}\right)^{2}+\lambda_{2}^{2} \kappa_{2}^{2}}\left(-\kappa_{2}+\lambda_{3} K+\lambda_{2} \sqrt{\kappa^{2}\left(\kappa_{1}-\kappa_{2}\right)^{2}-K^{2}}\right)}\right) .
$$

Then, we have also principal curvatures from the equations (12), (13), (15), (17), (28), (30) and (31)

$$
\kappa_{1}^{f}=\frac{-K\left(1-\lambda_{3} \kappa_{2}\right)+\lambda_{2} \kappa_{2} \sqrt{\kappa^{2}\left(\kappa_{1}-\kappa_{2}\right)^{2}-K^{2}}}{\sqrt{\left(1-\lambda_{3} \kappa_{2}\right)^{2}+\lambda_{2}^{2} \kappa_{2}^{2}}\left(\lambda_{3} K-\kappa_{2}+\lambda_{2} \sqrt{\kappa^{2}\left(\kappa_{1}-\kappa_{2}\right)^{2}-K^{2}}\right)},
$$

and from the equations (17), (29)

$$
\kappa_{2}^{f}=\frac{1}{R}=\frac{\kappa_{2}}{\sqrt{\left(1-\lambda_{3} \kappa_{2}\right)^{2}+\lambda_{2}^{2} \kappa_{2}^{2}}} .
$$

Thus, we can express the following theorems.

Theorem 2. Let $M$ be a tubular surface in $E^{3}$ and $M^{f}$ be surface at a constant distance from the edge of regression on $M$, under the condition that $\lambda_{1}=0$. Gauss and mean curvatures of $M^{f}$ are respectively,

$$
\begin{array}{r}
K^{f}=\frac{-K \kappa_{2}\left(1-\lambda_{3} \kappa_{2}\right)+\lambda_{2} \kappa_{2}^{2} \sqrt{\kappa^{2}\left(\kappa_{1}-\kappa_{2}\right)^{2}-K^{2}}}{\left(\left(1-\lambda_{3} \kappa_{2}\right)^{2}+\lambda_{2}^{2} \kappa_{2}^{2}\right)\left(\lambda_{3} K-\kappa_{2}+\lambda_{2} \sqrt{\kappa^{2}\left(\kappa_{1}-\kappa_{2}\right)^{2}-K^{2}}\right)}, \\
H^{f}=\frac{1}{2}\left(\frac{-K-\kappa_{2}^{2}+2 \kappa_{2}\left(\lambda_{3} K+\lambda_{2} \sqrt{\left.\kappa^{2}\left(\kappa_{1}-\kappa_{2}\right)^{2}-K^{2}\right)}\right.}{\sqrt{\left(1-\lambda_{3} \kappa_{2}\right)^{2}+\lambda_{2}^{2} \kappa_{2}^{2}}\left(-\kappa_{2}+\lambda_{3} K+\lambda_{2} \sqrt{\kappa^{2}\left(\kappa_{1}-\kappa_{2}\right)^{2}-K^{2}}\right)}\right),
\end{array}
$$

where $\kappa_{1}$ and $\kappa_{2}$ are principal curvatures of tubular surface $M$.

Theorem 3. Let $M$ be a tubular surface in $E^{3}$ and $M^{f}$ be surface at a constant distance from the edge of regression on $M$, under the condition that $\lambda_{1}=0$. In this case, principal curvatures of $M^{f}$ are

$$
\kappa_{1}^{f}=\frac{-K\left(1-\lambda_{3} \kappa_{2}\right)+\lambda_{2} \kappa_{2} \sqrt{\kappa^{2}\left(\kappa_{1}-\kappa_{2}\right)^{2}-K^{2}}}{\sqrt{\left(1-\lambda_{3} \kappa_{2}\right)^{2}+\lambda_{2}^{2} \kappa_{2}^{2}}\left(\lambda_{3} K-\kappa_{2}+\lambda_{2} \sqrt{\kappa^{2}\left(\kappa_{1}-\kappa_{2}\right)^{2}-K^{2}}\right)}
$$

and

$$
\kappa_{2}^{f}=\frac{1}{R}=\frac{\kappa_{2}}{\sqrt{\left(1-\lambda_{3} \kappa_{2}\right)^{2}+\lambda_{2}^{2} \kappa_{2}^{2}}} .
$$

Theorem 4. Let the pair of tubular surface $\left(M, M^{f}\right)$ be and the center curve $c$ of $M$ be given. In this case, parameter curves of $M^{f}$ are line of curvatures if and only if $c$ is planar.

Proof. We consider that tubular surface $M$ is given by parameterization

$$
\phi(s, \theta)=c(s)+r(\cos \theta N(s)+\sin \theta B(s)) .
$$


In this case, $M^{f}$ is given by the equation (18), for $\lambda_{1}=0$. If parameter curvatures are line of curvatures, $F=f=0$, where $F$ is coefficient of first fundamental form and $f$ is coefficient of second fundamental form. Then, $F=R^{2} \tau=0$ and $f=R \tau=0$, from the eq. (20) and (24). Since $R=\sqrt{\left(r-\lambda_{3}\right)^{2}+\lambda_{2}^{2}}, r \neq 0$ and $\lambda_{2} \neq 0, \lambda_{3} \neq 0$ and $R \neq 0, \tau=0$. Therefore, $c$ is planar.

On the contrary, if $c$ is planar, $\tau=0$. Hence, $F=f=0$. Thus, parameter curvatures are line of curvatures.

Theorem 5. Let the pair of tubular surface $\left(M, M^{f}\right)$ be and the center curve $c$ of $M$ be given. In this case, s-parameter curvatures of $M^{f}$ are given by $\psi(s, \gamma)$ are asymptotic curves if and only if curvatures of center curve $c$ satisfy the condition

$$
\frac{\tau^{2}}{\kappa}=\left(\frac{\left(r-\lambda_{3}\right) \cos \theta-\lambda_{2} \sin \theta}{\left(r-\lambda_{3}\right)^{2}+\lambda_{2}^{2}}\right)\left(1-\kappa\left(\left(r-\lambda_{3}\right) \cos \theta-\lambda_{2} \sin \theta\right)\right) .
$$

Proof. We consider that tubular surface $M$ is given by parameterization,

$$
\phi(s, \theta)=c(s)+r(\cos \theta N(s)+\sin \theta B(s)) .
$$

In this case, $M^{f}$ is given by the equation (18), for $\lambda_{1}=0$. If parameter curvatures are asymptotic curvatures, $e=g=0$, where $e, g$ are coefficients of second fundamental form. Then, if $s$-parameter curvatures of $M^{f}$ are asymptotic curves, from the equation (23), we have

$$
e=-\kappa \cos \gamma(1-R \kappa \cos \gamma)+R \tau^{2}=0 .
$$

Using the equations (12), (13), (15) and (17), for $\cos \gamma$, we obtain the following equation:

$$
\frac{\tau^{2}}{\kappa}=\left(\frac{\left(r-\lambda_{3}\right) \cos \theta-\lambda_{2} \sin \theta}{\left(r-\lambda_{3}\right)^{2}+\lambda_{2}^{2}}\right)\left(1-\kappa\left(\left(r-\lambda_{3}\right) \cos \theta-\lambda_{2} \sin \theta\right)\right) .
$$

This finish the proof.

Corollary 1. Let the pair of tubular and parallel surface $\left(M, M^{f}\right)$ be and the center curve c of $M$ be given. In this case, $s$ - parametercurvatures of $M^{f}$ is given by $\psi(s, \gamma)$ are asymptotic curves if and only if curvatures of center curve $c$ satisfy the condition

$$
\frac{\tau^{2}}{\kappa}=\left(\frac{\cos \theta\left(1-\kappa\left(r-\lambda_{3}\right) \cos \theta\right)}{r-\lambda_{3}}\right) .
$$

Proposition 1. Let the pair of tubular surface $\left(M, M^{f}\right)$ be and the center curve $c$ of $M$ be given. In this case, $\gamma$-parameter curvatures of $M^{f}$ are given by $\psi(s, \gamma)$ are not asymptotic curves.

Proof. If parameter curvatures are asymptotic curvature, $e=g=0$. From the equation (25), we can write $g=\left\langle\psi_{\gamma \gamma}, n^{f}\right\rangle=$ $R \neq 0$. Hence, $\gamma$-parameter curvatures are not asymptotic curves.

Theorem 6. Let the pair of tubular surface $\left(M, M^{f}\right)$ be and the center curve $c$ of $M$ be given. In this case, s-parameter curvatures of $M^{f}$ is given by $\psi(s, \gamma)$ are geodesic curves if and only if curvatures of center curve $c$ satisfy the condition

$$
\begin{gathered}
\sqrt{\left(r-\lambda_{3}\right)^{2}+\lambda_{2}^{2}}\left(\frac{r-\lambda_{3}}{\sqrt{\left(r-\lambda_{3}\right)^{2}+\lambda_{2}^{2}}} \cos \theta-\frac{\lambda_{2}}{\sqrt{\left(r-\lambda_{3}\right)^{2}+\lambda_{2}^{2}}} \sin \theta\right)^{2} \kappa^{2} \\
-2\left(\frac{r-\lambda_{3}}{\sqrt{\left(r-\lambda_{3}\right)^{2}+\lambda_{2}^{2}}} \cos \theta-\frac{\lambda_{2}}{\sqrt{\left(r-\lambda_{3}\right)^{2}+\lambda_{2}^{2}}} \sin \theta\right) \kappa+\sqrt{\left(r-\lambda_{3}\right)^{2}+\lambda_{2}^{2}} \tau^{2}=a,
\end{gathered}
$$

where $a$ is a constant. 
Proof. For a curve $\alpha$ on a surface $M$, condition of being geodesic is characterized by the $n \times \alpha^{\prime \prime}=0$. From the eq. (19) and (21), we get,

$$
n^{f} \times \psi_{s s}=\left(\kappa \sin \gamma-R \kappa^{2} \sin \gamma \cos \gamma-R \tau^{\prime}\right) T+\left(R \kappa^{\prime} \sin \gamma \cos \gamma-R \kappa \tau \sin ^{2} \gamma\right) N+\left(-R \kappa^{\prime} \cos ^{2} \gamma+R \kappa \tau \sin \gamma \cos \gamma\right) B
$$

Here, if $s$-parameter curvatures are geodesic curves, the following equation system is satisfied

$$
\begin{aligned}
\kappa \sin \gamma-R \kappa^{2} \sin \gamma \cos \gamma-R \tau^{\prime} & =0, \\
R \kappa^{\prime} \sin \gamma \cos \gamma-R \kappa \tau \sin ^{2} \gamma & =0, \\
-R \kappa^{\prime} \cos ^{2} \gamma+R \kappa \tau \sin \gamma \cos \gamma & =0 .
\end{aligned}
$$

From the solution of this equation system, we have,

$$
\kappa^{\prime} \cos \gamma-R \kappa \kappa^{\prime} \cos ^{2} \gamma-R \tau \tau^{\prime}=0
$$

and finally, we get

$$
R \kappa^{2} \cos ^{2} \gamma-2 \kappa \cos \gamma+R \tau^{2}=a(a \in \mathbb{R})
$$

If values of $R$ and $\cos \gamma$ in the above equation are written, the proof is completed.

Corollary 2. Let the pair of tubular and parallel surface $\left(M, M^{f}\right)$ be and the center curve cof $M$ be given. In this case, s-parameter curvatures of $M^{f}$ is given by $\psi(s, \gamma)$ are geodesic curves if and only if curvatures of center curve c satisfy the condition

$$
\left(r-\lambda_{3}\right) \cos ^{2} \theta \kappa^{2}-2 \cos \theta \kappa+\left(r-\lambda_{3}\right) \tau^{2}=a,
$$

where $a \in \mathbb{R}$.

\section{Conclusion}

The paper deals with tubular surface and surfaces at a constant distance from the edge of regression on a tubular surface in $E^{3}$. Firstly, we show that surface at a constant distance from the edge of regression on a tubular surface under the condition that $\lambda_{1}=0$ is also a tubular surface. Later, by calculating curvatures of these surfaces, we obtain some relationships between curvatures. Finally, we give the conditions geodesic curve, asymptotic curve and line of curvature of center curve $c$ of $M^{f}$.

\section{Acknowledgment}

The authors would like to thanks the referees for useful and improving comments.

\section{Competing interests}

The authors declare that they have no competing interests.

\section{Authors' contributions}

All authors have contributed to all parts of the article. All authors read and approved the final manuscript. 


\section{References}

[1] Ö. Tarakc1, Surfaces at a Constant Distance From The Edge of Regression on a Surface, PhD thesis, Ankara University Institute of Science, (2002), 101pp.

[2] Ö. Tarakcı and H.H. Hacısalihoğlu, Surfaces at a Constant Distance From The Edge of Regression on a Surface, Applied Mathematics and Computation, 155, (2004), 81-93.

[3] N. Aktan, A. Görgülü, E. Özüsağlam and C. Ekici, Conjugate Tangent Vectors and Asymptotic Directions for Surfaces at a Constant Distance From Edge of Regression on a Surface, IJPAM, 33, No. 1, (2006), 127-133.

[4] N. Aktan, E. Özüsağlam and A. Görgülü, The Euler Theorem and Dupin Indicatrix for Surfaces at a Constant Distance From Edge of Regression on a Surface, International Journal of Applied Mathematics\&Statistics, 14, No.S09, (2009), 37-43.

[5] D. Sağlam and Ö. Boyacioğlu Kalkan, Surfaces at a Constant Distance From The Edge of Regression on a Surface in $E_{1}^{3}$, Differential Geometry-Dynamical Systems, 12, (2010), 187-200.

[6] D. Sağlam and Ö. Boyacioğlu Kalkan, The Euler Theorem and Dupin Indicatrix for Surfaces at a Constant Distance From The Edge of Regression on a Surface in $E_{1}^{3}$, Matematicki Vesnik, 65, No.2, (2013), 242-249.

[7] D. Sağlam and Ö. Boyacığlu Kalkan, Conjugate Tangent Vectors and Asymptotic Directions for Surfaces at a Constant Distance From Edge of Regression on a Surface in $E_{1}^{3}$, Konuralp Journal of Mathematics, 2, No. 1, (2014), 24-35.

[8] S. Yurttançıkmaz and Ö. Tarakcı, The Relationship Between Focal Surfaces and Surfaces at a Constant Distance From The Edge of Regression On a Surface, Advances in Mathematical Physics, (2014), Article ID 397126.

[9] A. Çakmak and Ö. Tarakc1, The Image Curves on Surfaces at a Constant Distance from the Edge of Regression on a Surface of Revolution, International Journal of Mathematics and Computation, Vol. 27; No.1, (2016), 74-85.

[10] A. Çakmak and Ö. Tarakcı, Surface at a Constant Distance from the Edge of Regression on a Surface of Rotation in $E^{3}$, Applied Mathematical Sciences, Vol 10, no. 15, (2016), 707-719.

[11] H. Çayır, Some Notes on Lifts of Almost Paracontact Structures, American Review of Mathematics and Statistics, 3 (1), (2015), $52-60$.

[12] H. Çayır and K. Akda $\breve{g}$, Some notes on almost paracomplex structures associated with the diagonal lifts and operators on cotangent bundle, New Trends in Mathematical Sciences, 4 (4), (2016), 42-50.

[13] A.A. Salimov and H. Çayır, Some Notes On Almost Paracontact Structures, Comptes Rendus de 1'Acedemie Bulgare Des Sciences, 66 (3), (2013), 331-338.

[14] M.K. Karacan and Y. Yaylı, On the Geodesics of Tubular Surfaces in Minkowski 3-Space, Bulletin of the Malaysian Mathematical Sciences Society, (2), 31(1), (2008), 1-10.

[15] M. Dede, Tube surfaces in pseudo-Galilean space, International Journal of Geometric Methods in Modern Physics Vol. 13, No. 05,(2016), 1650056.

[16] S. Kızıltuğ and Y. Yayl1, Timelike tubes with Darboux frame in Minkowski 3-space International Journal of Physical Sciences, Vol. 8(1), (2013), pp. 31-36.

[17] M. K. Karacan and Y. Tuncer, Tubular surfaces of Weingarten types in Galilean and pseudo-Galilean, Bull. Math. Anal. Appl. 5, (2013), 87-100.

[18] A. Gray, Modern Differential Geometry of Curves and Surfaces with Mathematica, 2nd ed. Boca Raton, FL: CRC Press, (1997), pp. 207-209. 